\title{
ON MIDCONVEX FUNCTIONS WITH MIDCONCAVE BOUNDS
}

\author{
C. T. NG
}

(Communicated by R. Daniel Mauldin)

\begin{abstract}
A representation theorem for midconvex functions with midconcave upper bounds is obtained. It solves a problem posed by $\mathrm{K}$. Nikodem.
\end{abstract}

1. Introduction. A real-valued function $f$ defined on a convex subset $D$ of the Euclidean $n$-space $\mathbf{R}^{n}$ is midconvex if

$$
f\left(\frac{1}{2} x+\frac{1}{2} y\right) \leq \frac{1}{2}[f(x)+f(y)]
$$

for all $x, y$ in $D$. It is convex if $f(\lambda x+(1-\lambda) y) \leq \lambda f(x)+(1-\lambda) f(y)$ for all $x, y$ in $D$ and $\lambda$ in $[0,1]$. Midconcavity and concavity are defined by reversing the above inequalities. Midconvexity is frequently referred to as Jensen convexity, following the work of J. L. W. V. Jensen in 1905 and 1906.

There are many papers giving conditions under which midconvex functions are convex. A comprehensive review on this subject can be found in $[1,4]$. The following problem posed by K. Nikodem in [3] is of a different kind: Let $D$ be an open interval on $\mathbf{R}$. Let $f, g: D \rightarrow \mathbf{R}$ be midconvex and midconcave respectively, with $f(x) \leq g(x)$ for all $x$ in $D$. Do there exist functions $F, G: D \rightarrow \mathbf{R}, A: \mathbf{R} \rightarrow \mathbf{R}$, with $F$ convex, $G$ concave and $A$ additive such that $f=F+A$ and $g=G+A$ (while restricting $A$ to $D$ )?

The answer is positive. In the next section we present the result under a more general setting.

2. A representation theorem. We begin with some definitions and terminology. A function $A: \mathbf{R}^{n} \rightarrow \mathbf{R}$ is additive if $A(x+y)=A(x)+A(y)$ for all $x, y \in \mathbf{R}^{n}$. An affine function is the sum of an additive function and a constant. Let $f: D \rightarrow \mathbf{R}$ be defined on an open subset $D$ of $\mathbf{R}^{n}$. We say that $f$ is locally bounded from above by a midconcave (affine) function at a point $a \in D$ if for some convex neighborhood $N$ of $a$ in $D$, there exists a midconcave (affine, respectively) $g: N \rightarrow \mathbf{R}$ such that $f(x) \leq g(x)$ on $N$.

THEOREM. Let $f: D \rightarrow \mathbf{R}$ be defined on a nonvoid open convex subset of $\mathbf{R}^{n}$. The following statements are equivalent:

(1) $f$ is midconvex and is locally bounded from above by a midconcave function at some point $a$ in $D$.

(2) $f=F+A$ on $D$ for some convex $F: D \rightarrow \mathbf{R}$ and additive $A$.

Received by the editors July 24, 1986 and, in revised form, November 24, 1986.

1980 Mathematics Subject Classification (1985 Revision). Primary 26B25, 26B40; Secondary 39A10, 39C05.

Key words and phrases. Convexity, midconvexity, Cauchy functional equation.

Work supported by NSERC of Canada grant. 
(3) $f$ is midconvex and is locally bounded from above at each point a in $D$ by an affine function.

Proof. To show that (1) implies (2), let $f$ be midconvex, $N$ be a convex neighborhood of $a \in D, g: N \rightarrow \mathbf{R}$ be midconcave with the dominance $f \leq g$ on $N$. Without loss of generality we assume that $N$ is open, and for a moment focus our attention on $N$.

For each fixed $b \in \mathbf{R}^{n}$ we consider the functions

$$
f_{b}(x):=f(x)+f(b-x), \quad g_{b}(x):=g(x)+g(b-x)
$$

for all $x \in N$ with $b-x \in N$. Thus their domain is the open convex set $N \cap$ $(b-N)$ (which could be void). They inherit from $f$ and $g$ the properties: (i) $f_{b}$ is midconvex and $g_{b}$ is midconcave, and (ii) $f_{b} \leq g_{b}$. What is more is that they are (iii) symmetric about $b / 2$ in the sense that $f_{b}(x)=f_{b}(b-x)$ and $g_{b}(x)=g_{b}(b-x)$. This third property and (i) imply that $f_{b}$ and $g_{b}$ are minimized and maximized at $b / 2$ respectively. For example

$$
g_{b}(x)=g_{b}(b-x)=\left[g_{b}(x)+g_{b}(b-x)\right] / 2 \leq g_{b}[x / 2+(b-x) / 2]=g_{b}(b / 2)
$$

shows that $g_{b}$ is bounded from above by the constant $g_{b}(b / 2)$. In view of (ii), we obtain the boundedness of $f_{b}$ from above by the constant $g_{b}(b / 2)$. Since $f_{b}$ is midconvex, the boundedness from above leads to the conclusion that $f_{b}$ is continuous [4, Theorem 71C].

For each fixed $c \in \mathbf{R}^{n}$ we will establish the continuity of the difference $f(x+c)-$ $f(x)$ in the variable $x$ on the domain $N \cap(N-c)$. To this end, let $x_{0} \in N \cap(N-c)$ be arbitrarily given. We select arbitrarily a point $d \in N+x_{0}$. Then $x_{0}, x_{0}+c$ and $d-x_{0}$ are all in $N$. Since $N$ is open, there exists a neighborhood $V\left(x_{0}\right)$ of $x_{0}$ such that $x, x+c$ and $d-x$ are all in $N$ for every $x \in V\left(x_{0}\right)$. Thus every term in the identity

$$
f(x+c)-f(x)=[f(x+c)+f(d-x)]-[f(x)+f(d-x)]
$$

is defined whenever $x \in V\left(x_{0}\right)$. The function $[f(x+c)+f(d-x)]$ in $x$, being the composition of a translation $x \rightarrow x+c$ and the continuous $f_{d+c}$, is continuous at $x_{0}$. The function $[f(x)+f(d-x)]$ in $x$ is but $f_{d}$, and so is continuous at $x_{0}$. Thus the above identity yields the continuity of $f(x+c)-f(x)$ at $x=x_{0}$. As $x_{0}$ is arbitrary, this proves the continuity of $f(x+c)-f(x)$ in $x$ on $N \cap(N-c)$.

By a theorem of De Bruijn and J. H. B. Kemperman [2, Theorem 5.1], the continuity of the difference $f(x+c)-f(x)$ implies the existence of an additive function $A$ such that $F:=f-A$ is continuous on $N$. Hence $F$ is locally bounded at $a$.

We now return to $D$. The midconvexity of $f$ on $D$ implies the midconvexity of $F$ on $D$. The midconvexity of $F$ and its local boundedness at $a$ imply its convexity on $D$ [4, Theorem 71C]. This proves that $f$ is the sum of the convex $F$ and the additive $A$ on $D$.

To show that (2) implies (3), let $f=F+A$ be such a decomposition, and let $a \in D$ be arbitrarily given. Since $F$ is convex, it is continuous, and so must be bounded from above by some constant $\mu$ on some convex neighborhood $N$ of $a$. Then $f=F+A$ is bounded from above on $N$ by the affine function $\mu+A$.

As (3) trivially implies (1), the proof is complete. 
To address the problem by Nikodem, let $f, g: D \rightarrow \mathbf{R}$ be respectively midconvex and midconcave functions defined on a nonvoid open convex subset $D$ on $\mathbf{R}^{n}$, with $f \leq g$. Thus $f$ is locally bounded from above by $g$ at each point in $D$. By the above theorem, we can choose an additive $A$ such that $F:=f-A$ is convex (on $D$ ). With the same $A$ we consider $G:=g-A$. The midconcavity of $g$ implies that of $G$. Furthermore, from $f \leq g$ we get $F \leq G$. Since the convex $F$ is locally bounded from below (by constants), so is $G$. This proves that the midconcave $G$ is indeed concave [4, Theorem 71C].

\section{REFERENCES}

1. E. F. Beckenbach, Convex functions, Bull. Amer. Math. Soc. 54 (1948), 439-460.

2. J. H. B. Kemperman, A general functional equation, Trans. Amer. Math. Soc. 86 (1957), 28-56.

3. K. Nikodem, Problems and remarks (Proceedings of the International Conference on Functional Equations and Inequalities, May 27-June 2, 1984, Sielpia (Poland)), Wyż. Szkoła Ped. Krakow. Rocznik Nauk.-Dydakt. Prace Mat. 97 (1985).

4. A. Wayne Roberts and Dale E. Varberg, Convex functions, Pure and Appl. Math. Series, Academic Press, New York, 1973.

Department of Pure Mathematics, University of Waterloo, Waterloo, ONTARIO, CANADA N2L 3G1 